\title{
VARIABLES PSICOLÓGICAS IMPLICADAS EN EL DESEMPEÑO LABORAL DOCENTE
}

\author{
Manuel Torres V. ${ }^{1}$, Rosario Lajo \\ Universidad Nacional Mayor de San Marcos, Lima, Perú \\ (RECIBIDO EL 28/10/2008, ACEPTADO EL 11/12/2008)
}

\begin{abstract}
RESUMEN
El propósito del presente estudio es analizar las relaciones causales que pueden existir entre algunas variables psicológicas (Personalidad Tipo A, Afrontamiento al Estrés y Síndrome de burnout) y el desempeño laboral de los docentes universitarios de cinco facultades de medicina de Lima Metropolitana. Los instrumentos que se utilizaron fueron el Inventario de autorreporte de conducta tipo A de Blumenthal, COPE, el inventario de Burnout de Maslasch y la Prueba de desempeño laboral docente elaborada por Manuel Fernández Arata. Todos estos instrumentos fueron sometidos a los análisis respectivos para determinar su validez y confiabilidad.

La muestra estuvo constituida por 150 médicos docentes a quienes se les aplicó los cuestionarios. Los resultados indican que la Personalidad Tipo A, el Afrontamiento al Estrés y el Síndrome de Burnout influyen sobre su desempeño laboral, por lo que existe la necesidad de que las universidades desarrollen programas específicos que permitan a los docentes manejar de manera eficiente sus emociones.
\end{abstract}

Palabras clave: Emoción, Burnout, personalidad, estrés, desempeño laboral.

\begin{abstract}
The purpose of this study is to analyze the casual relations that can exist between some psychological variables (Personality Type A, Stress facing and Burnout Syndrome) and the labour performance of university teachers from five faculties of medicine of Lima Metropolitana. The instruments used were: Blumenthal's inventory of auto report of behaviour type A, COPE, Maslasch's Burnout inventory and the teacher's labour performance made by Manuel Fernández Arata. All these instruments were subjected to the respective analysis in order to determinate their validity and reliability.

The sample was constituted by 150 doctors teachers to whom the questionnaires were applied. The results show that Personality Type A, Stress Facing and Burnout Syndrome have influence over their labour performance, so is necessary that universities develop specific programmes that allow teachers manage their emotions in an efficient way.
\end{abstract}

Keywords: Emotion, Burnout, personality, stress, labour performance.

1 Docente Principal de la Facultad de Psicología de la UNMSM. E-mail: mtorresv@unmsm.edu.pe 


\section{INTRODUCCIÓN}

En un mundo donde la creciente profundización de las diferencias sociales, económicas y culturales entre las personas que viven en él, y caracterizado en simultáneo por el rápido conocimiento de esas diferencias a través de los medios de comunicación y las redes de información disponibles en nuestra sociedad mediática; en plena era de la globalización, la interdependencia económica, la posmodernidad, etc., afrontar los retos procedentes del significado educativo de estar juntos, de reconocer al otro en su diversidad, tratar de hacerlo desde una perspectiva dialógica, integradora, constituye, sin lugar a dudas, un magnífico empeño.

Sin embargo, en el mundo actual donde el uso masivo de la tecnología y el exceso de información lejos de reducir el trabajo lo acrecienta, se produce en el entorno laboral una acumulación de tareas -bajo la presión de los plazos de tiempo- que desencadena el estrés. La realidad es que se trabaja más y, de alguna manera, se está siempre en contacto con el centro laboral, todo ello a expensas del tiempo libre o personal. Aunque, si bien es cierto que un cierto nivel de estrés puede ser beneficioso para mantener el dinamismo en el trabajo, cuando este nivel se dispara se produce ese agotamiento emocional, de despersonalización y de reducción de la capacidad personal que ha dado en llamarse síndrome de burnout.

Lento pero, de manera progresiva, comenzarán a aparecer síntomas relativos a la situación psicológica de "estar quemado" o síndrome de burnout. Este síndrome se caracteriza por la disminución de la energía y la capacidad de concentración de la persona que se siente sobrepasada en todos los aspectos de la vida. Hace referencia a un "cansancio emocional que conlleva a la pérdida de motivación y eventualmente progresa hacia sentimientos de inadecuación y fracaso".

Esta patología es una de las principales causas del ausentismo laboral y se ha convertido en la fuente más importante para el sistema de salud y el ámbito laboral. Se ha comentado que los mayores determinantes del síndrome son la baja implicación laboral, el escaso nivel de autonomía, la indefinición de las tareas, el malestar físico, la elevada presión del trabajo, la ausencia de apoyo en la supervisión y la dirección, rutina y la burocratización.

Tras diversos estudios, se ha podido constatar cómo algunas de las características de la personalidad pueden llevar fácilmente a la persona sometida a un exceso de estrés a desarrollar un burnout. Las características son sensibilidad a los sentimientos y necesidades de los otros, dedicación al trabajo, idealismo, personalidad ansiosa y elevada autoexigencia. Por estas razones resulta importante desarrollar mecanismos de protección a la personalidad de los sujetos (estrategias de afrontamiento al estrés) que les permitan superar las dificultades y reducir los riesgos de desarrollar este síndrome.

El tratamiento de las enfermedades por estrés laboral deberá siempre dirigirse a erradicarlo a través de controlar los factores o las fuerzas causales del mismo. El criterio general que pretende curar la enfermedad en forma aislada mediante tratamiento paliativo de las alteraciones emocionales o reparación de las lesiones orgánicas es sumamente simplista, limitado y poco racional. Así, el tratamiento contra el estrés deberá ser preventivo y deberá lograrse ejerciendo las acciones necesarias para modificar los procesos causales. 
La prevención y atención del estrés laboral constituyen un gran reto, los criterios para contrarrestarlo deberán ser organizacionales y personales. Los médicos de salud en el trabajo y profesionales afines deben vigilar a sus pacientes y cuando sea posible a toda la organización con objeto de manejar el estrés en forma efectiva, aunque la participación del equipo de salud para efectuar cambios sustanciales con frecuencia es más difícil, pues los gerentes y empleadores generalmente buscan resolver el problema de los trabajadores en forma individual, pero rechazan la intervención en el origen del problema cuando esto implica la necesidad de cambios en el lugar de trabajo, por la posible disyuntiva entre la ganancia económica y el bienestar de los trabajadores.

El médico debe buscar anticipar la situación y aplicar medidas profilácticas efectivas. La prevención primaria es un objetivo primordial, las acciones eficaces han demostrado éxito económico en las empresas, al mejorar el estado de ánimo y el bienestar de los trabajadores disminuyendo de ese modo las enfermedades y el ausentismo, elevando la productividad y mejorando sustancialmente el desempeño y la calidad del trabajo.

A nivel personal, un aspecto fundamental en la prevención del estrés es la forma cómo los individuos pueden afrontar el estrés. En gran medida la diferencia entre individuos en cuanto a funcionamiento ante las situaciones de estrés se produce en las distintas formas en que cada uno le haga frente. Cuando hablamos de afrontamiento hacemos referencia a los esfuerzos tanto cognitivos como conductuales que realiza la persona para manejar el estrés psicológico, independientemente de sus resultados.

Siendo los médicos, profesionales muy expuestos a desarrollar esta patología, el problema se acrecienta grandemente cuando se dedican a la docencia universitaria como actividad complementaria o equivalente a su trabajo médico, por todo lo que implica hoy la actividad docente.

En los sistemas educativos de hoy el perfil del profesor ya no es el de un especialista titulado académicamente en un área determinada, sino el de un profesional que ocupa el rol de educador, un papel mucho más complejo que el del sistema anterior y que exige del docente la posesión de una mayor preparación pedagógica. Esta formación inicial incluye el manejo por parte del docente de un bagaje psicopedagógico que le hace conocedor de la psicología del alumnado. El conocimiento de la realidad social y afectiva del alumnado es clave en la creación de climas que favorezcan el aprendizaje y sustenten la intervención psicoeducativa pertinente.

Dentro de este microcontexto social, el clima idóneo para que se produzca una situación de enseñanza/ aprendizaje depende, en gran medida, de la calidad de las interacciones interpersonales de sus miembros. Es obvio que el alumno llega a la Universidad con una experiencia familiar y social que ha podido condicionar, positiva o negativamente, la actitud del mismo hacia el aprendizaje. No debemos olvidar que, principalmente en la Universidad es el medio social donde ocurren la mayor parte de sus actuaciones interpersonales.

Este "contexto de interacciones interpersonales" se convierte en un medio perfecto donde alcanzará la madurez emocional, suscitando así una serie de inquietudes intelectuales necesarias para el enriquecimiento y crecimiento personal del joven. Sin embargo, el profesor médico no siempre se encuentra en el aula con unas actitudes positivas ante la educación o ante sus pacientes. 
Es por esto que el médico debe recibir una preparación lo más integral posible que le permita no solo desarrollar los conocimientos y habilidades para realizar su trabajo, sino también debe recibir una preparación psicoemocional que le permita afrontar la presión laboral a la que se ve sometido diariamente.

\section{Problemas}

El presente estudio ha pretendido responder a las siguientes interrogantes de investigación:

- ¿Cuál es la influencia que tiene la personalidad tipo A, el afrontamiento al estrés y el síndrome de burnout, en el desempeño laboral de los médicos docentes de las Facultades de Medicina de Lima?

- $\quad$ ¿Qué relaciones existen entre la personalidad tipo A, el afrontamiento al estrés, el síndrome de Burnout y el desempeño laboral de los médicos docentes de las Facultades de Medicina de Lima?

- ¿Qué diferencias existen entre los médicos docentes de las Facultades de Medicina de Lima, respecto de la personalidad tipo A, el afrontamiento al estrés, el síndrome de burnout y el desempeño laboral?

- ¿Qué diferencias existen entre los médicos docentes considerando el género, respecto de la personalidad tipo A, el afrontamiento al estrés, el síndrome de burnout y el desempeño laboral?

- ¿ ¿Cuál es el nivel de burnout que presentan los médicos docentes de las Facultades de Medicina de Lima?

\section{Objetivos}

\section{General}

- Determinar la influencia que tiene la personalidad tipo A, el afrontamiento al estrés y el síndrome de burnout, en el desempeño laboral de los médicos docentes de las Facultades de Medicina de Lima.

\section{Específicos}

- Establecer las relaciones que existen entre la personalidad tipo A, el afrontamiento al estrés, el síndrome de burnout y el desempeño laboral de los médicos docentes de las Facultades de Medicina de Lima.

- Analizar las diferencias que existen entre los médicos docentes de las Facultades de Medicina de Lima, respecto de la personalidad tipo A, el afrontamiento al estrés, el síndrome de burnout y el desempeño laboral.

- Describir las diferencias que existen entre los médicos docentes considerando el género, respecto de la personalidad tipo $\mathrm{A}$, el afrontamiento al estrés, el síndrome de Burnout y el desempeño laboral. 
- Describir y analizar el nivel de burnout que presentan los médicos docentes de las Facultades de Medicina de Lima.

\section{Hipótesis}

\section{General}

- La personalidad tipo A, el afrontamiento al estrés y el síndrome de Burnout influyen significativamente en el desempeño laboral de los médicos docentes de las Facultades de Medicina de Lima.

\section{Específicos}

- Existen relaciones significativas entre la personalidad tipo A, el afrontamiento al estrés, el síndrome de burnout y el desempeño laboral de los médicos docentes de las Facultades de Medicina de Lima.

- Existen diferencias significativas entre los médicos docentes de las Facultades de Medicina de Lima, respecto de la personalidad tipo A, el afrontamiento al estrés, el síndrome de Burnout y el desempeño laboral.

- Existen diferencias significativas entre los médicos docentes considerando el género, respecto de la personalidad tipo A, el afrontamiento al estrés, el síndrome de Burnout y el desempeño laboral.

\section{Variables}

- Personalidad tipo A

- $\quad$ Afrontamiento al estrés

- $\quad$ Síndrome de burnout

- Desempeño laboral docente

\section{MÉTODO}

La presente investigación corresponde a una investigación de tipo descriptiva, de acuerdo a lo señalado por Sánchez y Reyes (2002), en la medida que se pretende describir y analizar sistemáticamente un conjunto de hechos (personalidad tipo A, afrontamiento al estrés y síndrome de burnout) relacionados causalmente con otro fenómeno (desempeño laboral docente), tal como se dan en el presente. Sin embargo la naturaleza causal de la presente investigación nos lleva a situarla dentro de los estudios ex post facto.

La investigación ex post facto es un tipo de investigación que se aplica cuando no se puede producir el fenómeno o no conviene hacerlo. Una característica esencial de la investigación ex post facto es que no se tiene control sobre la variable independiente, puesto que sus manifestaciones ya han ocurrido. Es decir, ha ocurrido un hecho (variable independiente) y se observan posteriormente los efectos en las variables dependientes. Es apropiado cuando el método experimental no es posible. Para Kerlinger (1985) "la investigación $e x$ 
post facto es una búsqueda sistemática empírica, en la cual el científico no tiene control directo sobre las variables independientes, porque ya acontecieron sus manifestaciones o por ser intrínsecamente no manipulables. Se hacen inferencias sobre las relaciones entre ellas, sin intervención directa, a partir de la variación concomitante de las variables independientes y dependientes".

\section{Diseño}

Para los efectos de la presente investigación, el diseño específico que utilizaremos es el diseño correlacional causal; Hernández Fernández y Baptista (2003) afirman que:

"Estos diseños describen relaciones entre dos o más variables en un momento determinado. Se trata también de descripciones, pero no de variables individuales sino de sus relaciones, sean estas puramente correlacionales o relaciones causales. En estos diseños lo que se mide es la relación entre variables en un tiempo determinado. Por lo tanto los diseños correlacionales pueden limitarse a establecer relaciones entre variables sin precisar sentido de causalidad o pueden pretender analizar relaciones de causalidad. Cuando se limitan a relaciones no causales, se fundamentan en hipótesis correlacionales y cuando buscan evaluar relaciones causales, se basan en hipótesis causales".

El análisis causal es una de las técnicas más recientes y más sofisticadas dentro de la corriente correlacional. El análisis discriminante, el análisis factorial, el análisis de escalas multidimensionales, el modelo de ecuaciones estructurales, etc. son ejemplos de análisis multivariables que parten de la correlación. Es por ello importante resaltar que, para este tipo de estudios, la estadística a utilizar resulta fundamental en tanto es la única que nos permite establecer las relaciones causales que pueden existir entre las variables.

\section{Composición de la muestra}

El diseño de la muestra es no probabilístico de tipo intencionado, en tanto fue el investigador quien definió el lugar de trabajo para la investigación y el número de la muestra. La muestra estuvo constituida por 150 médicos docentes (ver tabla N. ${ }^{\circ} 1$ ), con edades que fluctúan entre 16 y 27 años, siendo las de mayor proporción las que corresponden a los 19 años, 18 y 20 años de edad. También se aprecia que la muestra está conformada mayoritariamente por mujeres $71,9 \%$, frente al $28,1 \%$ de los varones.

Tabla N. ${ }^{0}$ 1. Composición general de la muestra.

\begin{tabular}{lll}
\hline \multicolumn{1}{c}{ Sexo } & F & \% \\
\hline Masculino & 44 & 28,1 \\
Femenino & 106 & 71,9 \\
\hline Universidad & & \\
\hline UNMSM & 30 & 20,0 \\
UPCH & 30 & 20,0 \\
UNFV & 30 & 20,0 \\
USMP & 30 & 20,0 \\
URP & 30 & 20,0 \\
\hline
\end{tabular}




\section{Instrumentos}

Los instrumentos que se utilizaron para recolectar los datos fueron: Inventario de autorreporte de conducta tipo A de Blumenthal, Inventario de afrontamiento al estrés COPE, Inventario de Burnout de Maslasch y Prueba de desempeño laboral docente de Manuel Fernández. El análisis psicométrico del Inventario de autorreporte de conducta tipo A de Blumenthal indica que sus dos escalas alcanzan un alfa de cronbach de 0,87 y 0,80 respectivamente, por lo que se considera que la prueba es confiable

El análisis psicométrico del inventario de afrontamiento al estrés COPE indica que sus escalas alcanzan alfa de cronbach que fluctúan entre 0,70 y 0,79 por lo que se considera que la prueba es confiable.

El análisis psicométrico del inventario de Burnout de Maslasch indica que sus escalas alcanzan alfa de cronbach que fluctúan entre 0,78 y 0,92 por lo que se considera que la prueba es confiable.

El análisis psicométrico del inventario de desempeño laboral docente indica que sus escalas alcanzan alfa de cronbach que fluctúan entre 0,70 y 0,88 por lo que se considera que la prueba es confiable.

Los análisis de la Validez de los instrumentos, realizado a través del Análisis factorial Exploratorio nos muestran que las pruebas presentan validez de Constructo.

\section{RESULTADOS}

En lo que concierne al análisis de la forma de la distribución de los puntajes realizado a través del test de bondad de ajuste a la curva normal de Kolmogorov-Smirnov, indica que en las mediciones de las variables estudiadas se obtienen coeficientes K-S Z que no son estadísticamente significativos, concluyendo que se presentan adecuadas aproximaciones a la curva normal, por lo que es factible utilizar, para el análisis de los datos, estadísticas paramétricas.

\section{Contraste de hipótesis}

Con la finalidad de analizar la influencia de las variables Independientes (Personalidad tipo A de Blumenthal, afrontamiento al estrés COPE, síndrome de Burnout), sobre la variable dependiente conformada por una variable latente (desempeño laboral docente) se realizó el análisis del Modelo de Ecuaciones Estructurales.

El modelo presentado en la tabla N. ${ }^{\circ}$ 2, alcanza un chi-cuadrado de 13,36 el cual no es estadísticamente significativo, por lo que se puede concluir que el modelo es adecuado. El análisis estadístico multivariado del modelo propuesto en comparación con el modelo saturado, donde todas las variables están relacionadas entre sí, y el modelo Independiente, donde todas las variables son independientes entre sí, refleja que el primero resulta ser el más eficiente, pues presenta un chi-cuadrado menor $\left(X^{2}=13,36\right.$, G.L. $\left.=8, \mathrm{p}=, 100\right)$, así como una menor proporción entre chi-cuadrado y grados de libertad $(1,67)$. 
Tabla N. 2. Análisis de ecuaciones estructurales.

\begin{tabular}{lccc}
\hline & Modelo Propuesto & Modelo Saturado & Modelo Independiente \\
\hline Chi-Cuadrado & $\mathbf{1 3 , 3 6}$ & $\mathbf{0 , 0 0}$ & $\mathbf{4 2 0 , 4 1}$ \\
\hline G.L. & 8 & 0 & 36 \\
P & 100 & & 0,000 \\
Parámetros & 37 & 45 & 9 \\
$X^{2} /$ G.L. & 1,67 & & \\
RMR & 0,03 & 0,00 & 0,38 \\
GFI & 0,97 & 1,00 & 0,47 \\
AGFI & 0,84 & & 0,332 \\
\hline N = 150 & & &
\end{tabular}

Además se encuentra que el Índice de Bondad de Ajuste (GFI) alcanza un valor adecuado $(0,97)$, así como un índice de Bondad de Ajuste Corregido (AGFI) que también es aceptable $(0,84)$, notándose además que el índice de residuales medias cuadradas (RMR) alcanza un valor de 0,03 .

- Variables Independientes

- Estilo de afrontamiento enfocado en el problema (estilo 1)

- Estilo de afrontamiento enfocado en la emoción (estilo 2)

- Otros estilos de afrontamiento (estilo 3)

- Personalidad tipo a (tipo a)

- Personalidad tipo b (tipo b)

- Variables Intervinientes

- Despersonalización (DESPER)

- Cansancio emocional (CANEMO)

- Realización personal (REALPER)

- Variable Dependiente

- Total Desempeño Docente (TOTALDES)

El análisis de los resultados, tomando en cuenta las ponderaciones estandarizadas a fin de facilitar la interpretación de los resultados, indica que en el gráfico se aprecian los siguientes hallazgos:

- $\quad$ Estilo 1, ejerce un efecto indirecto sobre el desempeño docente pues su efecto es producido a través del cansancio emocional y la despersonalización

- $\quad$ Estilo 2, ejerce un efecto indirecto sobre el desempeño docente y este es básicamente a través de la despersonalización. 
Figura N. ${ }^{\circ}$ 1. Modelo de ecuaciones estructurales.

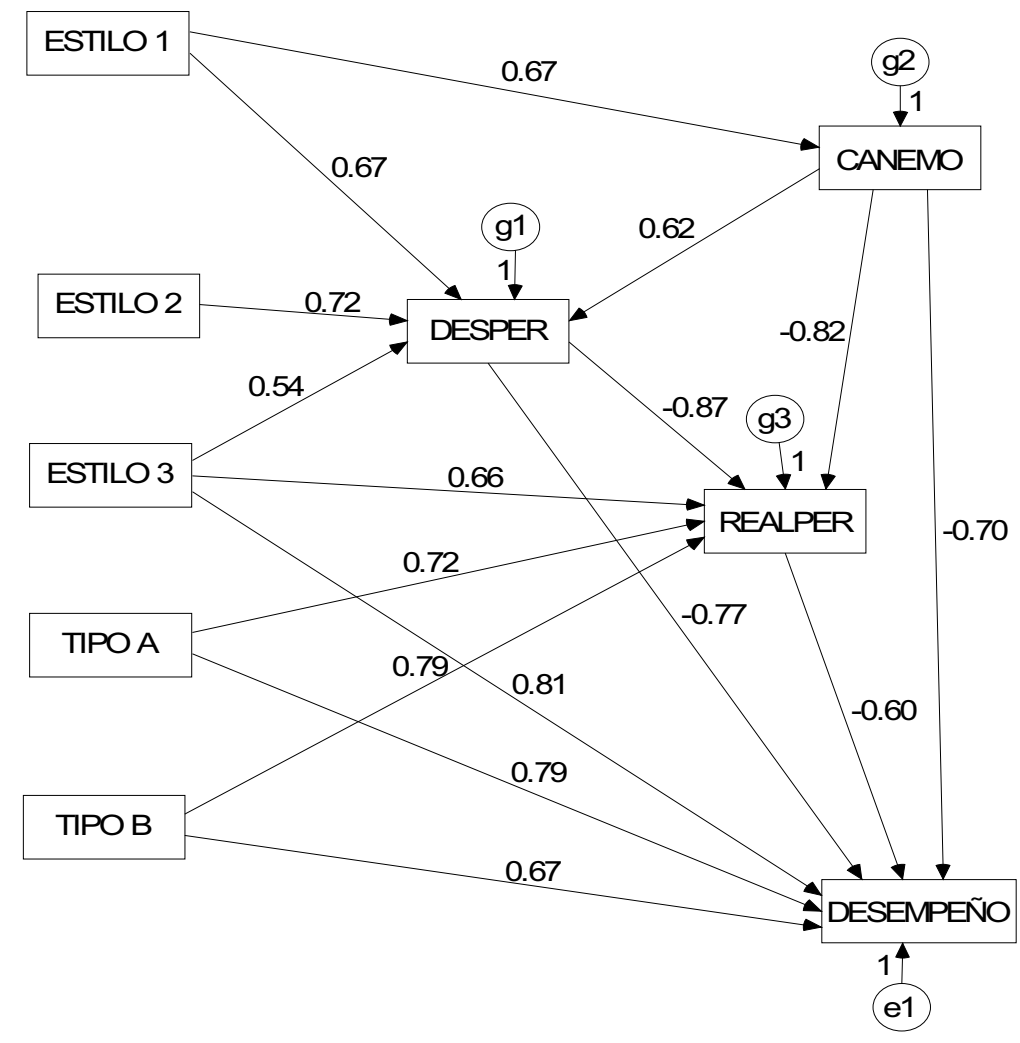

- $\quad$ Estilo 3, ejerce un efecto positivo directo sobre el desempeño docente y a la vez presenta efectos indirectos por medio de la despersonalización y la realización personal.

- $\quad$ Tipo A, ejerce un efecto positivo directo y también un efecto indirecto a través de la realización personal.

- Tipo B, ejerce un efecto positivo directo y además un efecto indirecto a través de la realización personal.

- Las variables que conforman el burnout presentan un efecto mediador, pues reciben la influencia de otras variables; sin embargo su efecto es negativo en el caso del cansancio emocional y la despersonalización, mientras que en la realización personal el efecto es positivo. 


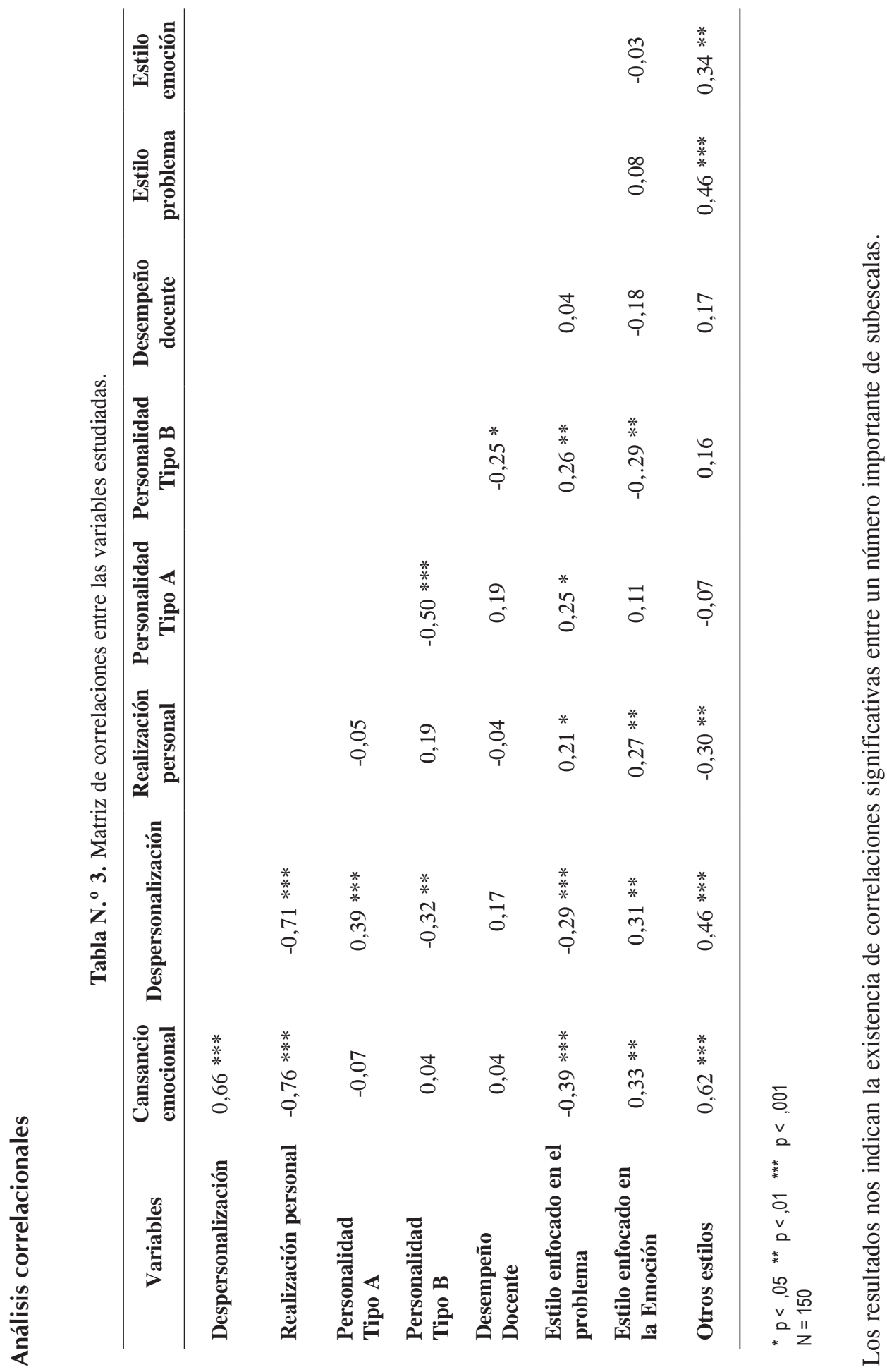




\section{Análisis comparativos}

Tabla N. ${ }^{4}$ 4. Comparación de los Tipos de Afrontamiento del COPE según sexo.

\begin{tabular}{|c|c|c|c|c|c|}
\hline \multirow{2}{*}{ VARIABLE } & \multicolumn{2}{|c|}{$\begin{array}{l}\text { Hombre } \\
\mathrm{N}=106\end{array}$} & \multicolumn{2}{|c|}{$\begin{array}{c}\text { Mujer } \\
\mathbf{N}=\mathbf{4 4} \\
\end{array}$} & \multirow[t]{2}{*}{$\mathbf{T}$} \\
\hline & $\mathbf{M}$ & D.E. & $\mathbf{M}$ & D.E. & \\
\hline Afrontamiento activo & 2,69 & 0,63 & 2,67 & 0,60 & 0,16 \\
\hline Planificación & 2,99 & 0,73 & 2,90 & 0,71 & 0,58 \\
\hline $\begin{array}{l}\text { Supresión de actividades } \\
\text { competentes }\end{array}$ & 2,79 & 0,57 & 2,62 & 0,53 & 1,37 \\
\hline Postergación del afrontamiento & 2,64 & 0,60 & 2,53 & 0,51 & 0,88 \\
\hline $\begin{array}{l}\text { Búsqueda de apoyo social por } \\
\text { razones instrumentales }\end{array}$ & 2,49 & 0.77 & 2,26 & 0,78 & 1,37 \\
\hline $\begin{array}{l}\text { Búsqueda de apoyo social por } \\
\text { razones emocionales }\end{array}$ & 2,19 & 0,66 & 2,15 & 0,70 & 0,21 \\
\hline $\begin{array}{l}\text { Reinterpretación positiva y } \\
\text { crecimiento }\end{array}$ & 2,94 & 0,61 & 3,00 & 0,69 & $-0,43$ \\
\hline Aceptación & 2,69 & 0,57 & 2,68 & 0,59 & 0,10 \\
\hline Acudir a la religión & 2,49 & 0,67 & 2,11 & 0,59 & $2,61 *$ \\
\hline Enfocar y liberar emociones & 2.49 & 0,78 & 2,68 & 0,67 & $-1,18$ \\
\hline Negación & 2.08 & 0,70 & 2,02 & 0,65 & 0,42 \\
\hline Desentendimiento conductual & 2.04 & 0,81 & 2,16 & 0,81 & $-0,72$ \\
\hline Desentendimiento mental & 2.25 & 0,71 & 2,17 & 0,63 & 0,49 \\
\hline
\end{tabular}

${ }^{*} p<, 05$

El análisis de las diferencias de los puntajes del COPE según sexo permite observar que sólo existen diferencias significativas en el tipo de Afrontamiento de Acudir a la religión $(\mathrm{T}=2,61, \mathrm{p}<, 05)$ notándose que los hombres $(\mathrm{M}=2,49$, D.E. $=0,67)$ alcanzan puntajes más elevados que las mujeres $(\mathrm{M}=2,11$, D.E. $=0,59)$. 
Tabla N. ${ }^{\circ}$ 5. Comparación de los Estilos de Afrontamiento del COPE según sexo.

\begin{tabular}{lcccccc}
\hline \multirow{2}{*}{\multicolumn{1}{c}{ VARIABLE }} & \multicolumn{2}{c}{ Hombre } & \multicolumn{2}{c}{ Mujer } \\
& \multicolumn{2}{c}{$\mathbf{N = \mathbf { 1 0 6 }}$} & \multicolumn{2}{c}{$\mathbf{N = \mathbf { 4 4 }}$} & \multirow{2}{*}{$\mathbf{T}$} \\
\cline { 2 - 5 } & $\mathbf{M}$ & $\mathbf{D . E}$ & $\mathbf{M}$ & \multicolumn{1}{c}{$\mathbf{D . E .}$} & \\
\hline Enfocado en el problema & 2,72 & 0,39 & 2,59 & 0,28 & 1,59 \\
Enfocado en la emoción & 2,48 & 0,33 & 2,39 & 0,28 & 1,21 \\
Otros estilos de afrontamiento & 2,26 & 0,61 & 2,34 & 0,46 & 0,66 \\
\hline
\end{tabular}

${ }^{*} p<, 05$

El análisis de los estilos de afrontamiento permite observar que no existen diferencias estadísticas significativas.

Tabla N. ${ }^{\circ}$ 6. Comparación de las áreas del MBI de Maslach según sexo.

\begin{tabular}{lccccc}
\hline \multirow{2}{*}{ VARIABLE } & \multicolumn{2}{c}{ Hombre } & \multicolumn{2}{c}{ Mujer } & \multirow{2}{*}{ T } \\
\cline { 2 - 5 } & \multicolumn{2}{c}{$\mathbf{N}=\mathbf{1 0 6}$} & \multicolumn{2}{c}{$\mathbf{N}=\mathbf{4 4}$} & \\
\cline { 2 - 5 } Cansancio emocional & 2,21 & 1,29 & 2,26 & 1,23 & 0,17 \\
Despersonalización & 1,86 & 1,87 & 1,02 & 1,59 & $2,13 *$ \\
Realización personal & 4,90 & 0,89 & 5,05 & 0,84 & 0,75 \\
\hline
\end{tabular}

${ }^{*} p<, 05$

El análisis de las áreas del MBI de Maslasch indica que existen diferencias estadísticas significativas en la Despersonalización $(\mathrm{T}=2,131, \mathrm{p}<, 05)$ notándose que los hombres $(\mathrm{M}=1,86$, D.E. $=1,87)$ alcanzan puntajes más elevados que las mujeres $(\mathrm{M}=1,02$, D.E. $=1,59)$.

Tabla N. ${ }^{0}$ 7. Comparación de la Personalidad Tipo A y B según sexo.

\begin{tabular}{cccccc}
\hline \multirow{2}{*}{ VARIABLE } & \multicolumn{2}{c}{ Hombre } & \multicolumn{2}{c}{ Mujer } \\
& \multicolumn{2}{c}{$\mathbf{N = \mathbf { 1 0 6 }}$} & \multicolumn{2}{c}{$\mathbf{N = \mathbf { 4 4 }}$} & \multirow{2}{*}{$\mathbf{T}$} \\
\cline { 2 - 5 } & $\mathbf{M}$ & $\mathbf{D . E}$ & $\mathbf{M}$ & $\mathbf{D . E .}$ & \\
\hline Conducta Tipo A & 4,77 & 0,83 & 4,19 & 0,97 & $2,97 * *$ \\
Conducta Tipo B & 2,83 & 1,10 & 3,61 & 1,11 & $-3,23 * *$ \\
\hline
\end{tabular}

${ }^{* *} p<, 01$ 
La revisión de los tipo de personalidad indica que existen diferencias significativas en la Personalidad Tipos A $(\mathrm{T}=2.97, \mathrm{p}<.01)$ notándose que los hombres $(\mathrm{M}=4.77$, D. E. $=0.83)$ alcanzan puntajes más elevados que las mujeres $(\mathrm{M}=4.19$, D.E. $=0.97)$. Además se observa que también existen diferencias en la Personalidad Tipo B $(\mathrm{T}=2.83$, $\mathrm{p}<.01)$ notándose que las Mujeres $(\mathrm{M}=3.61$, D. E. $=1.11)$ presentan puntajes más elevados que los hombres $(\mathrm{M}=2.83$, D.E. $=1.10)$.

Tabla N. ${ }^{\circ}$ 8. Comparación del desempeño docente según sexo.

\begin{tabular}{|c|c|c|c|c|c|}
\hline \multirow{2}{*}{ VARIABLE } & \multicolumn{2}{|c|}{$\begin{array}{l}\text { Hombre } \\
\mathbf{N}=106\end{array}$} & \multicolumn{2}{|c|}{$\begin{array}{c}\text { Mujer } \\
\mathrm{N}=44 \\
\end{array}$} & \multirow{2}{*}{$\mathbf{T}$} \\
\hline & $\mathbf{M}$ & D.E. & $\mathbf{M}$ & D.E. & \\
\hline Evaluación del desempeño en el aula & 5,72 & 0,66 & 5,90 & 0,53 & $-1,38$ \\
\hline $\begin{array}{l}\text { Autoevaluación de las labores en el } \\
\text { ámbito escolar }\end{array}$ & 3,57 & 1,14 & 3,06 & 0,97 & $2,11 *$ \\
\hline Interacción con los alumnos & 4,89 & 0,72 & 4,94 & 0,75 & $-0,33$ \\
\hline Desempeño docente total & 4,72 & 0,29 & 4,64 & 0,23 & 1,45 \\
\hline
\end{tabular}

${ }^{* *} p<, 01{ }^{* * *} p<, 001$

La comparación por desempeño docente indica que sólo existen diferencias significativas en la autoevaluación de las labores en el ámbito escolar $(\mathrm{T}=2,11, \mathrm{p}<, 05)$ notándose que los hombres $(\mathrm{M}=3,57, \mathrm{D}$. E. = 1,14) alcanzan puntajes más altos que las mujeres $(\mathrm{M}=3,06, \mathrm{D} . \mathrm{E} .=0,97)$.

Tabla N. 9. Comparación de los tipos de afrontamiento del COPE según universidad de procedencia.

\begin{tabular}{|c|c|c|c|c|c|c|}
\hline Tipo de Afrontamiento & $\begin{array}{c}\text { UMSM } \\
\mathbf{N}=\mathbf{3 0} \\
\mathbf{M}\end{array}$ & $\begin{array}{c}\text { UPCH } \\
\mathbf{N}=\mathbf{3 0} \\
\mathbf{M}\end{array}$ & $\begin{array}{c}\text { UNFV } \\
\mathbf{N}=\mathbf{3 0} \\
\mathbf{M}\end{array}$ & $\begin{array}{c}\text { USMP } \\
\mathbf{N}=\mathbf{3 0} \\
\mathbf{M}\end{array}$ & $\begin{array}{c}\text { URP } \\
\mathbf{N}=\mathbf{3 0} \\
\mathbf{M}\end{array}$ & $\mathbf{F}$ \\
\hline Afrontamiento activo & 2,78 & 2,70 & 2,75 & $2,98 \mathrm{a}$ & $2,24 \mathrm{a}$ & $4,44 * *$ \\
\hline Planificación & 3,01 & 2,83 & 3,03 & 3,31 & 2,64 & 2,58 \\
\hline Supresión de actividades competentes & 2,98 & 2,73 & 2,75 & 2,65 & 2,60 & 1,35 \\
\hline Postergación del afrontamiento & 2,75 & 2,46 & 2,55 & 2,66 & 2,60 & 0,72 \\
\hline $\begin{array}{l}\text { Búsqueda de apoyo social por razones } \\
\text { instrumentales }\end{array}$ & 2,33 & 2,46 & 2,44 & 2,45 & 2,45 & 0,10 \\
\hline $\begin{array}{l}\text { Búsqueda de apoyo social por razones } \\
\text { emocionales }\end{array}$ & 2,06 & 2,23 & 2,23 & 2,00 & 2,38 & 0,98 \\
\hline Reinterpretación positiva y crecimiento & 2,98 & 3,13 & 2,83 & 3,04 & 2,83 & 0,87 \\
\hline Aceptación & 2,90 & 2,55 & 2,50 & 2,79 & 2,71 & 1,73 \\
\hline Acudir a la religión & 2,40 & 2,78 & 2,30 & 2,78 & 2,64 & 1,07 \\
\hline Enfocar y liberar emociones & 2,67 & 2,70 & 2,49 & 2,13 & 2,74 & 2,38 \\
\hline Negación & 2,10 & 2,20 & 2,04 & 1,74 & 2,24 & 1,73 \\
\hline Desentendimiento conductual & 2,15 & $2,35 \mathrm{a}$ & 2,00 & $1,46 \mathrm{ab}$ & $2,40 \mathrm{~b}$ & $5,09 * *$ \\
\hline Desentendimiento mental & 2,26 & $2,44 \mathrm{a}$ & 2,23 & $1,76 \mathrm{ab}$ & $2,44 \mathrm{~b}$ & $3,62 * *$ \\
\hline
\end{tabular}




\section{Diferencias significativas por pares a través de la prueba de Scheffé: $a, b$}

Los resultados del análisis de varianza de los tipos de afrontamiento indican que existen diferencias estadísticas significativas según la universidad de procedencia en los siguientes aspectos:

Afrontamiento Activo ( $\mathrm{F}=4,44 \mathrm{p}<, 001)$ notándose que los participantes de la USMP $(\mathrm{M}=2,98)$ superan a los participantes de la URP $(\mathrm{M}=2,24)$.

Desentendimiento Conductual $(\mathrm{F}=5,09 \mathrm{p}<, 001)$ notándose que los participantes de la URP $(\mathrm{M}=2,40)$ y de la UPCH $(\mathrm{M}=2,35)$ superan a los participantes de la USMP $(\mathrm{M}=1,46)$.

Desentendimiento Mental $(\mathrm{F}=3,62 \mathrm{p}<, 001)$ notándose que los participantes de la URP $(\mathrm{M}=2,44)$ y la UPCH $(\mathrm{M}=2,44)$ superan a los participantes de la USMP $(\mathrm{M}=1,76)$.

Tabla N. ${ }^{\circ}$ 10. Comparación de los estilos de afrontamiento según universidad de procedencia.

\begin{tabular}{lcccccc}
\hline \multirow{3}{*}{ Estilos de afrontamiento } & UMSM & UPCH & UNFV & USMP & URP & \\
& $\mathbf{N = 3 0}$ & $\mathbf{N}=\mathbf{3 0}$ & $\mathbf{N = 3 0}$ & $\mathbf{N = 3 0}$ & $\mathbf{N = 3 0}$ & \multirow{2}{*}{$\mathbf{F}$} \\
\cline { 2 - 6 } & $\mathbf{M}$ & $\mathbf{M}$ & $\mathbf{M}$ & $\mathbf{M}$ & $\mathbf{M}$ & \\
\hline Enfocado en el problema & 2.77 & 2.64 & 2.70 & 2.81 & 2.51 & 2.33 \\
Enfocado en la emoción & 2.49 & 2.48 & 2.38 & 2.37 & 2.56 & 1.30 \\
Otros estilos de afrontamiento & $2.36 \mathrm{a}$ & $2.50 \mathrm{~b}$ & 2.24 & $1.78 \mathrm{abc}$ & $2.53 \mathrm{c}$ & $6.86 * *$ \\
\hline
\end{tabular}

** $p<, 01$

G. L. $1=2 \quad$ G. L. $2=92$

\section{Diferencias significativas por pares a través de la prueba de Scheffé: a, b}

Los resultados del análisis de varianza de los estilos de afrontamiento indican que existen diferencias estadísticas significativas en otros estilos de ofrontamiento $(\mathrm{F}=6,86 \mathrm{p}<$ ,001), notándose que los participantes de la URP $(\mathrm{M}=2,53)$, la UNMSM $(\mathrm{M}=2,36)$ y la UPCH $(\mathrm{M}=2,50)$ superan a los colegios estatales $(\mathrm{M}=1,78)$.

Tabla N. ${ }^{\circ}$ 11. Comparación de las áreas del MBI de Maslasch según universidad de procedencia.

\begin{tabular}{|c|c|c|c|c|c|c|}
\hline \multirow[t]{2}{*}{ Variable } & $\begin{array}{l}\text { UMSM } \\
\mathbf{N}=\mathbf{3 0} \\
\end{array}$ & $\begin{array}{c}\text { UPCH } \\
\mathbf{N}=\mathbf{3 0} \\
\end{array}$ & $\begin{array}{c}\text { UNFV } \\
\mathbf{N}=\mathbf{3 0} \\
\end{array}$ & $\begin{array}{c}\text { USMP } \\
\mathbf{N}=\mathbf{3 0} \\
\end{array}$ & $\begin{array}{c}\text { URP } \\
\mathbf{N}=\mathbf{3 0}\end{array}$ & $\mathbf{F}$ \\
\hline & $\mathbf{M}$ & $\mathbf{M}$ & $\mathbf{M}$ & M & M & \\
\hline Cansancio emocional & 2,45 & 2,49 & 2,11 & $1,40 \mathrm{a}$ & $2,67 \mathrm{a}$ & $3,46 * *$ \\
\hline Despersonalización & 1,88 & 1,52 & 1,26 & $0,77 \mathrm{a}$ & $2,67 \mathrm{a}$ & $3,32 *$ \\
\hline Realización personal & 4,99 & 4,81 & 4,83 & 5,30 & 4,78 & 1,21 \\
\hline
\end{tabular}

** $p<, 05 * * * 0<0,01$

G. L. $1=2 \quad$ G. L. $2=92$ 


\section{Diferencias significativas por pares a través de la prueba de Scheffé: $a, b$}

Los resultados del análisis de varianza de las áreas del MBI de Maslasch, permiten observar que existen diferencias estadísticas significativas en:

Cansancio emocional ( $\mathrm{F}=3,46 \mathrm{p}<, 001)$ notándose que los participantes de la URP $(\mathrm{M}=2,67)$ superan a los participantes de la USMP $(\mathrm{M}=1,40)$.

Despersonalización $(\mathrm{F}=3,32 \mathrm{p}<.01)$, notándose que los participantes de la URP $(\mathrm{M}=2,67)$ superan a los participantes de la USMP $(\mathrm{M}=0,77)$.

Tabla N. ${ }^{0}$ 12. Comparación de los tipos de personalidad A y B según universidad de procedencia.

\begin{tabular}{ccccccc}
\hline \multirow{2}{*}{ Variable } & UMSM & UPCH & UNFV & USMP & URP & \\
& $\mathbf{N = 3 0}$ & $\mathbf{N = 3 0}$ & $\mathbf{N = 3 0}$ & $\mathbf{N = 3 0}$ & $\mathbf{N = 3 0}$ & \multirow{2}{*}{$\mathbf{F}$} \\
\cline { 2 - 6 } & $\mathbf{M}$ & $\mathbf{M}$ & $\mathbf{M}$ & $\mathbf{M}$ & $\mathbf{M}$ & \\
\hline Personalidad Tipo A & 4,84 & 4,45 & 4,59 & 4,45 & 4,67 & 0,66 \\
Personalidad Tipo B & 3,00 & 3,25 & 2,91 & 3,08 & 3,03 & 0,23 \\
\hline
\end{tabular}

G. L. $1=2 \quad$ G. L. $2=92$

Los resultados del análisis de varianza de los tipos de personalidad A y B no presentan diferencias estadísticas significativas.

Tabla N. ${ }^{\circ}$ 13. Comparación del desempeño docente según universidad de procedencia.

\begin{tabular}{lcccccc}
\hline \multirow{2}{*}{ Variable } & $\begin{array}{c}\text { UMSM } \\
\mathbf{N = 3 0}\end{array}$ & $\begin{array}{c}\text { UPCH } \\
\mathbf{N}=\mathbf{3 0}\end{array}$ & $\begin{array}{c}\text { UNFV } \\
\mathbf{N}=\mathbf{3 0}\end{array}$ & $\begin{array}{c}\text { USMP } \\
\mathbf{N}=\mathbf{3 0}\end{array}$ & $\begin{array}{c}\text { URP } \\
\mathbf{N}=\mathbf{3 0}\end{array}$ & $\mathbf{F}$ \\
\cline { 2 - 7 } & $\mathbf{M}$ & $\mathbf{M}$ & $\mathbf{M}$ & $\mathbf{M}$ & $\mathbf{M}$ & \\
\hline $\begin{array}{l}\text { Evaluación del desempeño } \\
\text { en el aula }\end{array}$ & 5,82 & 5,65 & 5,85 & 6,05 & 5,49 & 2,43 \\
$\begin{array}{l}\text { Autoevaluación de las } \\
\text { labores en el ámbito escolar }\end{array}$ & 3,34 & 3,38 & 3,25 & $2,98 \mathrm{a}$ & $4,14 \mathrm{a}$ & $3,31 * *$ \\
$\begin{array}{l}\text { Interacción con los alumnos } \\
\text { Desempeño Docente Total }\end{array}$ & 4,96 & 5,03 & 4,79 & 5,01 & 4,72 & 0,71 \\
\hline
\end{tabular}

** $p<.05{ }^{* * *} p<0,01$

$\begin{array}{lll}\text { G. L. } 1=2 & \text { G. L. } 2=92\end{array}$

\section{Diferencias significativas por pares a través de la prueba de Scheffé: $a, b$}

Los resultados del análisis de varianza de las áreas del desempeño docente indican que existen diferencias estadísticas significativas en la autoevaluación de las labores en el ámbito escolar $(\mathrm{F}=3,31 \mathrm{p}<, 001)$ notándose que los participantes de la URP $(\mathrm{M}=$ $4,14)$ superan a los participantes de la USMP $(\mathrm{M}=2,98)$. 


\section{Análisis descriptivos}

Tabla N. ${ }^{0}$ 14. Nivel de burnout en la muestra estudiada.

\begin{tabular}{lcc}
\hline \multicolumn{1}{c}{ Burnout } & F & $\mathbf{\%}$ \\
\hline Bajo & 68 & 46,0 \\
Medio & 15 & 10,0 \\
Alto & 66 & 44,00 \\
\hline Total & 150 & $100,00 \%$ \\
\hline
\end{tabular}

La reclasificación de los resultados de la tabla anterior en 3 niveles, indica que el 46,0 $\%$ de los casos alcanzan niveles bajos, en tanto que el 44,00\% presenta niveles altos y el $10,0 \%$ valores medios. Estos hallazgos corroboran que los sujetos de la muestra en más del 54,0 \% han desarrollado el síndrome de burnout.

\section{DISCUSIÓN}

De acuerdo a los resultados obtenidos tenemos que la hipótesis general de investigación ha sido básicamente aceptada, es decir se ha demostrado que tanto la personalidad tipo A como el afrontamiento al estrés y el síndrome de Burnout influyen significativamente en el desempeño laboral de los médicos docentes, afectando positiva o negativamente su rendimiento; en este aspecto el efecto del burnout parece ser muy limitante para el trabajo del médico docente. Este efecto lo podemos entender como el agotamiento, desgaste o fatiga psicológica en el trabajo, genéricamente como una forma de aflicción producida por un sobreesfuerzo en el trabajo, que se manifiesta en severas pérdidas de energía, desrealización profesional y en un descenso considerable en calidad y cantidad de rendimiento.

Las cifras de prevalencia encontradas son bastantes altas y coincidentes con los resultados encontrados en otros países. Igualmente se ratifica lo propuesto por Maslach en 1981 en su investigación, cuando plantea que aquellas personas que por naturaleza o especiales características de su trabajo debían mantener un contacto directo y continuo con la gente, tras un período de tiempo largo, algunas de ellas acaban sufriendo un importante desgaste personal y laboral.

Debemos considerar que el médico está muy expuesto al sufrimiento, no sólo del paciente sino también de él mismo. El paciente sufre cada día, pero también el médico, y esto supone un gran desgaste psíquico (Maslach, 1982) que trata de compensar con conductas altruistas que pueden agravar su estabilidad emocional. Surge entonces el tedio, y sus actividades cotidianas ya no despiertan el entusiasmo y el interés de otros tiempos. En este sentido, es interesante reflexionar sobre el hecho de que menospreciar las actividades cotidianas puede producir sentimientos de depresión, agobio y resentimiento.

Por estas razones, señalamos nuestra preocupación por el alto índice de burnout que encontramos en los resultados de la presente investigación y que, sin duda, están atentando 
contra el trabajo profesional y docente de los médicos. De allí la necesidad de encontrar alternativas viables que faciliten la actividad de los médicos y les permitan regular mejor su salud y bienestar personal.

Por otra parte es importante añotar que si bien las diferencias encontradas en los resultados comparativos realizados entre los médicos de acuerdo al sexo, se presentan sólo en la escala de Despersonalización $(\mathrm{T}=2,131, \mathrm{p}<, 05)$, notándose que los hombres $(\mathrm{M}=1,86$, D.E. $=1,87)$ alcanzan puntajes más elevados que las mujeres $(\mathrm{M}=1,02, \mathrm{D} . \mathrm{E} .=1,59)$, es importante resaltar que los hombres parecen estar más expuestos a desarrollar este síndrome.

Finalmente, se debe señalar la que tiene la evaluación de los docentes como una forma de fomentar y favorecer el perfeccionamiento del profesorado, asimismo identificar las cualidades que conforman a un buen profesor para, a partir de ahí, generar políticas educativas que coadyuven a su generalización.

Estos resultados nos permiten postular que los nuevos sistemas educativos deben considerar la necesidad de introducir cursos y/o programas de entrenamiento que puedan contribuir a mejorar el bienestar emocional de los profesores. Es necesario fomentar los valores personales y familiares, aprovechar cualquier momento de convivencia con la familia y con los compañeros de trabajo. Las soluciones son preventivas y terapéuticas a nivel individual, grupal y organizacional. Entre las medidas preventivas, tenemos la de evitar que la enfermedad o el daño llegue; si la enfermedad ya se instauró, habrá que controlarla, tratarla, pedir apoyo y evitar complicaciones. Cuanto más pronto se instaure las medidas preventivas, la evolución o curación será una realidad.

\section{CONCLUSIONES}

1. Los resultados muestran que la Personalidad tipo A, el Afrontamiento al estrés y el Síndrome de burnout, influyen sobre el desempeño laboral de los médicos docentes.

2. Respecto de los análisis correlaciones, se ha encontrado la existencia de correlaciones significativas, en la mayoría de los casos, entre las variables estudiadas y sus respectivas subescalas.

3. Las diferencias encontradas entre los médicos docentes de las Facultades de Medicina de Lima, respecto de las variables en estudio, se presentan en los siguientes casos: Afrontamiento al estrés: afrontamiento activo, desentendimiento conductual y desentendimiento mental y otros estilos de afrontamiento. Burnout: cansancio emocional y despersonalización. Desempeño laboral docente: autoevaluación de las labores en el ámbito escolar.

4. Se ha comprobado la existencia de diferencias significativas sólo en los casos de Afrontamiento de acudir a la religión, despersonalización, personalidad tipo a y autoevaluación de las labores en el ámbito escolar. En todos estos casos los hombres han alcanzado valores más altos que las mujeres. Solo en el caso de Personalidad tipo B las mujeres presentan puntajes más elevados que los hombres. 
5. Los niveles de burnout que presentan los médicos docentes de las Facultades de Medicina de Lima es del $44 \%$ en el nivel alto y $10 \%$ en el nivel medio.

\section{REFERENCIAS BIBLIOGRÁFICAS}

1. Bisquerra, R. (1989). Introducción conceptual al análisis multivariable. Barcelona: PPU.

2. Buendía J, Ramos F. (2001). Empleo, estrés y salud. Madrid: Ediciones Pirámides.

3. First, M.B., y Pincus, H.A. (1996). DSM IV Manual de diagnóstico diferencial. Barcelona: Masson S.A.

4. Gil-Monte PR, Peiró JM. (1997). Desgaste psíquico en el trabajo: El síndrome de quemarse. Madrid: Síntesis.

5. Goleman D. (1997). La salud emocional. Barcelona: Kairos S. A.

6. Heller, Agnes (1980). Teoría de los sentimientos. Barcelona: Ed. Fontamara.

7. Hernández, Fernández y Baptista. (2006). Metodología de la Investigación. México: Ed. Mc Graw Hill.

8. Lazarus y Folkman, S. (1986). Estrés y procesos cognitivos. Barcelona: Martínez Roca.

9. Mayor, L. (comp.) (1988). Psicología de la emoción. Valencia: Promolibro.

10. Maslach, Christina. (1982). Enciclopedia de salud y seguridad en el trabajo. Ginebra: Editorial Organización Internacional del Trabajo,

11. Organización Mundial de la Salud. (2000). Salud ocupacional: Éticamente correcta, económicamente adecuada. Ginebra: Organización Mundial de la Salud (OMS).

12. Ortony, A., y Collins, A. (1996). La estructura cognitiva de las emociones. Madrid: Siglo veintiuno.

13. Pelechaño, V. y De Miguel, A. (1994). Estrés, personalidad y salud. Valencia: Editorial Alfaplus

14. Rodríguez M.J. (1995). Psicología social de la salud. Madrid: Síntesis.

15. Sánchez-Cánovas, J. (1988). “Estilos cognitivos, afrontamiento y psicología del Yo”. Boletín de Psicología N. ${ }^{\circ} 19$.

16. Sánchez, H. y Reyes, C. (2002). Metodología y diseños en la investigación científica. Lima: Universidad Ricardo Palma, Editorial Universitaria.

17. Taylor S.E. (1991). Seamos optimistas. Ilusiones positivas. Barcelona: Ediciones Martínez Roca. 